\title{
Vegetative Incompatibility and Cytoplasmic Infection in Fungi
}

\author{
By C. E. CATEN \\ Department of Genetics, University of Birmingham, Birmingham, B15 2TT
}

(Accepted for publication 29 March 1972 )

\begin{abstract}
SUMMARY
The effect of vegetative (heterokaryon) incompatibility on the transfer of a suppressive cytoplasmically determined condition, vegetative death, from carrier to normal strains of Aspergillus amstelodami has been investigated. Cytoplasmic transfer was reduced to I $5 \%$ by vegetative incompatibility compared with $100 \%$ transfer in compatible combinations. Successful transfer in incompatible combinations involved donors and recipients whose incompatibility was determined by a single gene, and transfer was completely prevented between strains differing for more than one incompatibility gene. These results support the hypothesis that vegetative incompatibility serves as a cellular defence mechanism against genetic infection by stopping the spread of viruses and other suppressive cytoplasmic determinants from strain to strain in nature. Vegetative incompatibility is likely to be important in determining the specificity of virus-host interactions in fungi.
\end{abstract}

\section{INTRODUCTION}

Two types of potentially harmful cytoplasmic inclusions possessing genetic continuity and hence capable of infection occur in fungal mycelia. These are (i) defective, mutant derivatives of essential cytoplasmic genetic elements which have acquired the ability to suppress their normal counterparts; and (ii) viruses. 'Vegetative death' in Aspergillus glaucus (Jinks, I956, I959), 'senescence' in Podospora anserina (Marcou, I96I) and the 'stopper' phenotypes in Neurospora crassa (Bertrand, McDougall \& Pittenger, 1968) are examples of the former which arose spontaneously during prolonged vegetative culture. Recent reports indicate that RNA viruses occur commonly in fungi (Hollings, 1962; Banks et al. I968; Hollings \& Stone, 1969; Border et al. 1972). While this division into mutants of normal cell components and supernumerary genetic elements can be made in theory, it may in practice be difficult to distinguish between them (Hollings \& Stone, 1969). Thus, although infectivity is normally required as a criterion for a virus, infection of virus-free strains by cell-free extracts containing virus-like particles has proved difficult to obtain, and it seems likely that the natural method of transmission of both viruses and mutant cytoplasms is via hyphal anastomosis and heterokaryon formation (Hollings \& Stone, I969; Lhoas, I97I). The ability of fungal mycelia to fuse and exchange protoplasms will therefore be important factors determining the spread of cytoplasmic genetic infections in natural fungal populations.

When independent strains of the same species of many Ascomycetes and Fungi Imperfecti are mixed, heterokaryon formation is prevented by systems of heterokaryon or vegetative incompatibility (Caten \& Jinks, 1966; Esser \& Kuenen, 1967; Fincham \& Day, 197I). Genetic studies in Neurospora crassa (Garnjobst, I953, I955; Holloway, 1955) and Podospora anserina (Esser, I97I) have shown that incompatibility is determined by heterozygosity at one or more specific incompatibility loci. The presence of different alleles at these loci 
in the anastomosing strains leads to the rapid degeneration and death of the fused cells following protoplasmic mixing (Garnjobst \& Wilson, 1956). Vegetative incompatibility was first revealed and has subsequently been studied almost entirely through its inhibitory effects on nuclear transfer and heterokaryon formation. Its consequences for cytoplasmic exchange have not been examined. In view of the important implications for the spread of suppressive cytoplasmic mutants and viruses in fungal populations, the effect of vegetative incompatibility on the transmission of an infectious cytoplasmic agent has been investigated directly.

\section{METHODS}

The system. Strains of Aspergillus amstelodami carrying the degenerative condition 'vegetative death' were used as donors in mixed cultures with compatible and incompatible, normal (i.e. non-vegetative death) recipients of the same species. Vegetative death (Vgd) is determined by a cytoplasmic genetic element which is transferred to a normal strain $\left(\mathrm{Vgd}^{+}\right)$ in an infectious manner following heterokaryon formation (Jinks, I956, I959). Electron microscopy of sectioned hyphae and mycelial homogenates of a vegetative death strain has failed to reveal virus-like particles ( $R$. Crosse, personal communication), and the nature of the determinant is unknown. Vegetative death is particularly suitable as a cytoplasmic marker since it is highly suppressive and has a marked effect upon the phenotype of the recipient strain, making it unnecessary to resort to polyacrylamide gel electrophoresis or electron microscopy to detect transfer (Lhoas, 1971).

Vegetative (heterokaryon) incompatibility was first reported in Aspergillus amstelodami by Jones (1965). Recent investigations of its genetic control (C. E. Caten, unpublished) indicate that it is determined by an allelic mechanism similar to that in Neurospora crassa and Podospora anserina (Garnjobst, 1953, 1955; Esser, 1971).

Strains. Vegetative death arose spontaneously in 1970 in Aspergillus amstelodami, strain $37 / 6$, which is a pyridoxine auxotroph isolated from wild-type 37 following treatment with $N$-methyl- $N^{\prime}$-nitro- $N$-nitrosoguanidine. The wild-type has remained normal. This source of vegetative death was therefore independent of that of Jinks (1956, 1959) but phenotypically very similar to it.

In one experiment (Expt I) $37 / 6 \mathrm{Vgd}$ was used as the donor in mixed cultures with a single compatible (202w) and two incompatible recipients $(36 / 23 \mathrm{w}$ and $\mathrm{I} / 57)$. A second experiment (Expt 2) involved the same donor with 202w, 36/23w and three other compatible and incompatible strains as recipients. The nine recipients used in these two experiments were all independent and represented a range of background genotypes (Table $2 a$ ). All five incompatible recipients differed from $37 / 6 \mathrm{Vgd}$ at more than one incompatibility locus. In a third experiment (Expt 3) a culture of $37 \mathrm{w}$ (a white conidial colour mutant of 37 ) to which vegetative death had been transferred in Expt 2 was used as the donor. The recipients were I I compatible and 9 incompatible progeny from a cross which was segregating for a single pair of alleles (hetAr:hetA2) determining heterokaryon compatibility with this donor (Table $2 b$ ). Thus in this experiment the incompatible combinations differed from the compatible solely in their heterozygosity at the het $A$ locus (incompatible = hetAI/het A2, compatible $=$ het $A I /$ het $A I$ ). All other segregating alleles should be distributed among the two types of recipient at random and hence will not bias the comparison of the compatible and incompatible combinations.

The nuclei of donor and recipient strains were distinguished both by spore colour and auxotrophic markers in Expts I and 2, but only by spore colour in Expt 3.

Media and culture methods. For morphological comparisons and determinations of 
(a) Mixed cultures

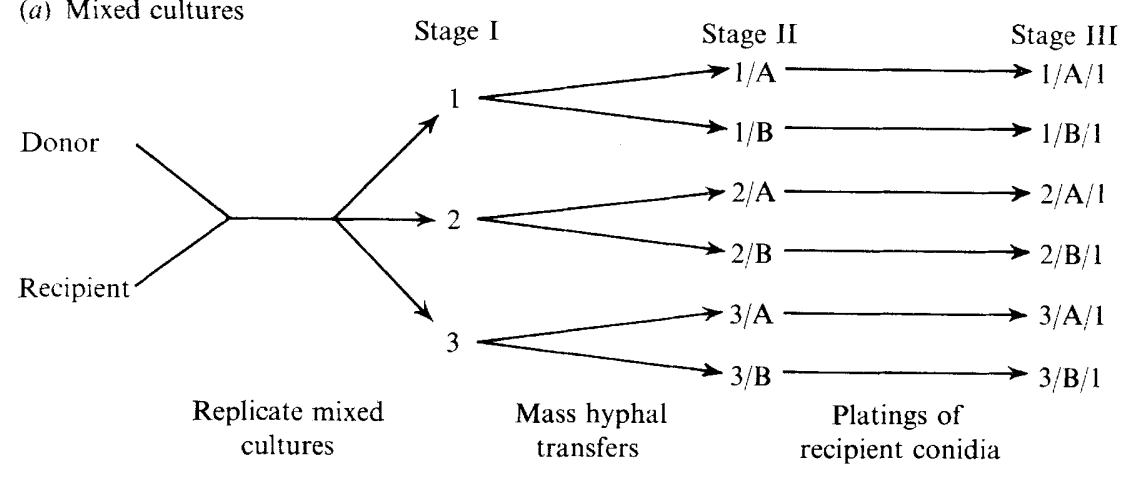

(b) Controls

As for mixed cultures, except donor or recipient grown alone

Fig. I. Summary of the experimental procedure for mixed cultures and controls.

colony size Czapek agar with $20 \%$ (w/v) sucrose $(20 \% \mathrm{CZ})$ was used. For rapid growth and conidiation $2 \%$ malt extract agar + sodium chloride at $75 \mathrm{~g} / 1$ (MTS) was used. Colony size reduction was obtained by adding sodium deoxycholate (Koch-Light Laboratories Ltd, Colnbrook, Buckinghamshire) to MTS (MTSD) at 0.4 to $0.6 \mathrm{mg} / \mathrm{ml}$. All media were supplemented throughout with the nutrients required to support growth of the strains used. Incubation was at $30^{\circ} \mathrm{C}$.

Experimental procedure. A number of independent mixed cultures (stage I) of each donor/ recipient combination was established by the adjacent point inoculation of conidia from the two strains on MTS medium. After incubation for 6 days, two or three independent replicates of each combination which showed a good mixture of the strains were analysed for the transfer of vegetative death. Two, three and two replicate donor/recipient combinations were tested in Expts I, 2 and 3 respectively. For analysis, two $\mathrm{I} \mathrm{mm}^{2}$ hyphal blocks (A and $\mathrm{B}$ ) were taken from a region of the culture where donor and recipient were intimately mixed and transferred to $20 \% \mathrm{CZ}$ plates, incubated for 5 days and the diameters of the resulting colonies (stage II) measured. To detect vegetative death in the recipient strains a single sample of recipient conidia, identified by their colour, was taken from each stage II colony with a fine glass needle and plated on MTSD. The size of the conidial sample was kept constant at a level which experience had shown to give Ioo to 300 colonies if taken from a normal strain. The chances of detecting vegetative death were maximized by deliberately sampling from areas showing reduced growth. The MTSD plates were examined after 5 or 6 days of incubation when colony formation was complete (stage III cultures). This whole procedure is summarized in Fig. I.

As controls for the spontaneous occurrence of vegetative death, each recipient strain was inoculated alone on to MTS in replicate and the resulting colonies analysed in the same way as the mixed cultures. If vegetative death occurred spontaneously in a strain, then the mixed cultures involving this strain as recipient were discarded. Furthermore to demonstrate the phenotypic effects of vegetative death on colony morphology and conidial germination, the donor strain was treated in the same way. 

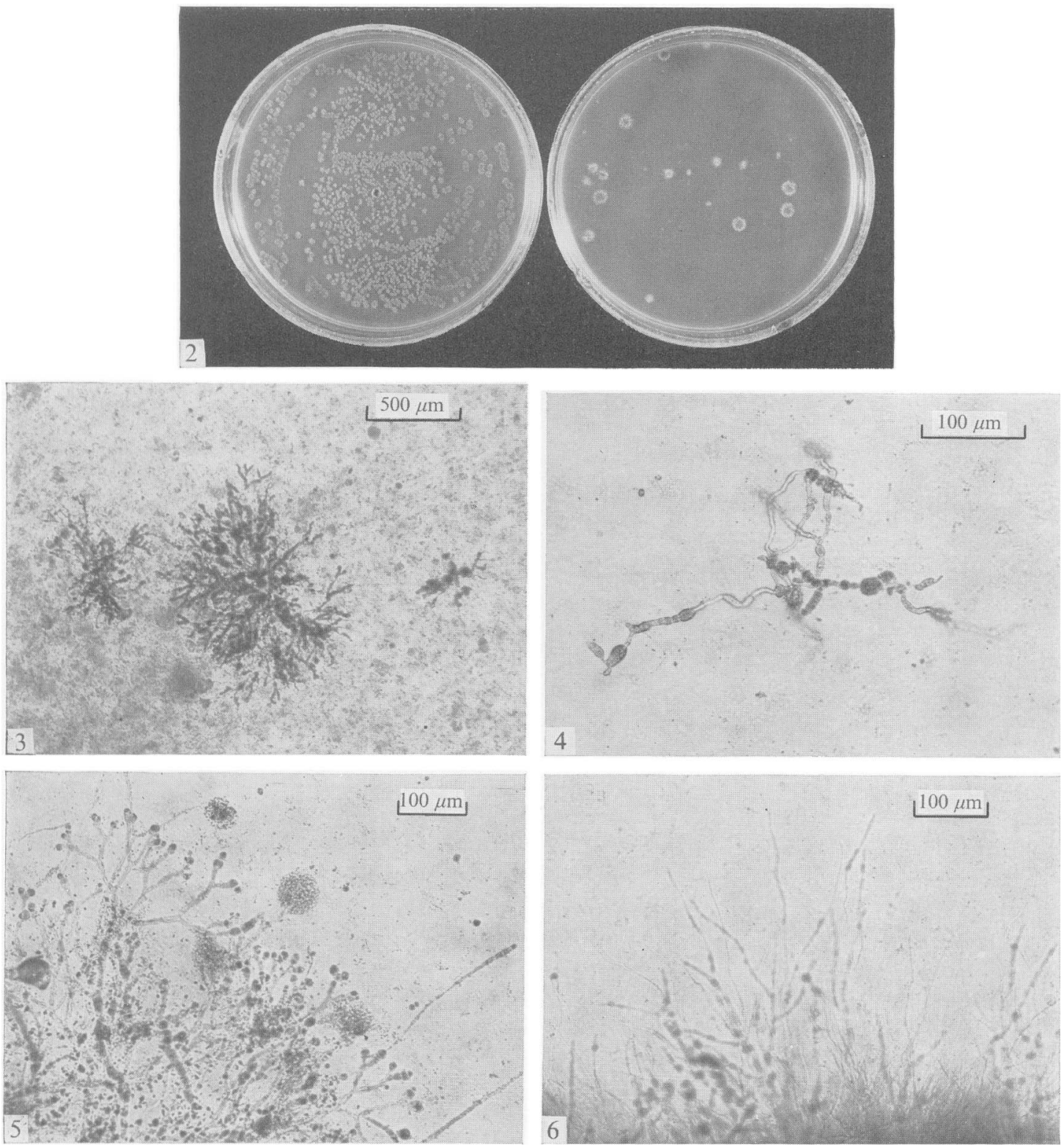

Fig. 2. Cultures derived from platings on to MTSD of equivalent samples of conidia from wild-type 37 on the left, and the vegetative-death-carrying strain $37 / 6 \mathrm{Vgd}$ on the right. Note the low viability of conidia from $37 / 6 \mathrm{Vgd}$ and the heterogeneity of the colonies produced.

Fig. 3 to 5. Detail of colonies from the culture of $37 / 6 \mathrm{Vgd}$ in Fig. 2.

Fig. 3. Three micro-colonies.

Fig. 4. A single micro-colony. Note the swollen distorted hyphae, and the presence of empty hyphae.

Fig. 5. The margin of one of the larger colonies which although near normal in size shows marked symptoms of vegetative death. Note the enlarged, much branched hyphae which are swelling and bursting at their tips.

Fig. 6. The margin of a colony of wild-type 37 from the culture in Fig. 2. Compare the fine, relatively unbranched hyphae with those in Fig. 5. 


\section{RESULTS}

Phenotypic effects of vegetative death. The effects of vegetative death on stage II and stage III colonies were shown by controls in which the donor was transferred alone, and were identical for both donors. Three of six mass hyphal transfers from $37 / 6 \mathrm{Vgd}$ failed to grow, while the rest produced the slow growing colonies with irregular 'moth-eaten' margins and poor conidiation characteristic of vegetative death (Table I $a$ ). Conidial platings from these colonies to give stage III cultures revealed that the majority of conidia were incapable of producing normal colonies (Fig. 2 and Table $\mathrm{I} b$ ) due to death of the colony at any stage during development (Table I $c$ ). Many conidia failed to germinate, others germinated but produced swollen, distorted germlings which ceased to develop further (Fig. 3, 4), and others developed to give a visible colony but with the irregular margins and low conidiation characteristic of the mass hyphal transfer colonies (Fig. 5). Thus conidial platings from vegetative-death strains gave a mosaic of all these abnormal micro-colony types plus the occasional apparently normal colony. Such cultures were very distinct from the abundant, uniform highly conidial colonies produced by comparable platings from vegetative-death-free strains (Fig. 2). The reduction in conidial viability and production of dying micro-colonies are characteristic features of vegetative death and were used as the diagnostic criteria.

Effects of mixed culture. All stage II cultures consisted principally of the recipient strain, the donor being restricted to heterokaryotic areas in the compatible combinations or small patches in the incompatible. Of eight stage II colonies of the compatible recipient $202 \mathrm{~W}$ all except one showed vegetative death symptoms and as a result the mean colony diameter was markedly less than that of the control colonies, all of which were completely normal (Table I $a$ ). In contrast none of the stage II colonies of $36 / 23 \mathrm{~W}$ had irregular margins and reduced conidiation, although their diameter was also significantly less than the controls. The reduction in size, however, was far less than with the compatible recipient (Table $\mathrm{r} a$ ).

The occurrence of symptoms in the stage II colonies does not necessarily reflect transfer of vegetative death to the recipient, since they may result from the presence of donor mycelium in the mixed culture. Transfer is confirmed, however, by the stage III cultures which were established purely from recipient conidia. For $202 \mathrm{w}$, seven of the eight cultures contained dying micro-colonies and only two produced any normal colonies, while the controls were completely free of vegetative death and produced many normal colonies (Table $\mathrm{I} b$ and $c$ ). As at stage II the appearance of the cultures of $36 / 23 \mathrm{w}$ contrasted with those for $202 \mathrm{w}$. None of the platings of $36 / 23 \mathrm{w}$ from the mixed cultures produced any dying microcolonies and the number of normal colonies was not significantly different from those of the controls (Table $\mathrm{I} b$ and $c$ ).

The detailed results for these two recipients illustrate the two patterns of response to culture with a vegetative-death-carrying strain observed. In one, mixed culture led to a characteristic series of phenotypic changes in the recipient which closely paralleled those of the donor, while in the other, it had little or no effect and development of the recipient was essentially normal. One or the other of these two clearly distinguishable responses was obtained in all 63 independent mixed cultures examined and consequently each mixture could be classified according to whether transfer occurred or not. This qualitative distinction is used in presenting the rest of the results.

Comparison of compatible and incompatible combinations. Whether the association between successful transfer and heterokaryon compatibility on the one hand, and failure of transfer and incompatibility on the other is a general phenomenon was determined by 


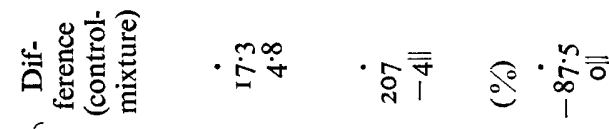

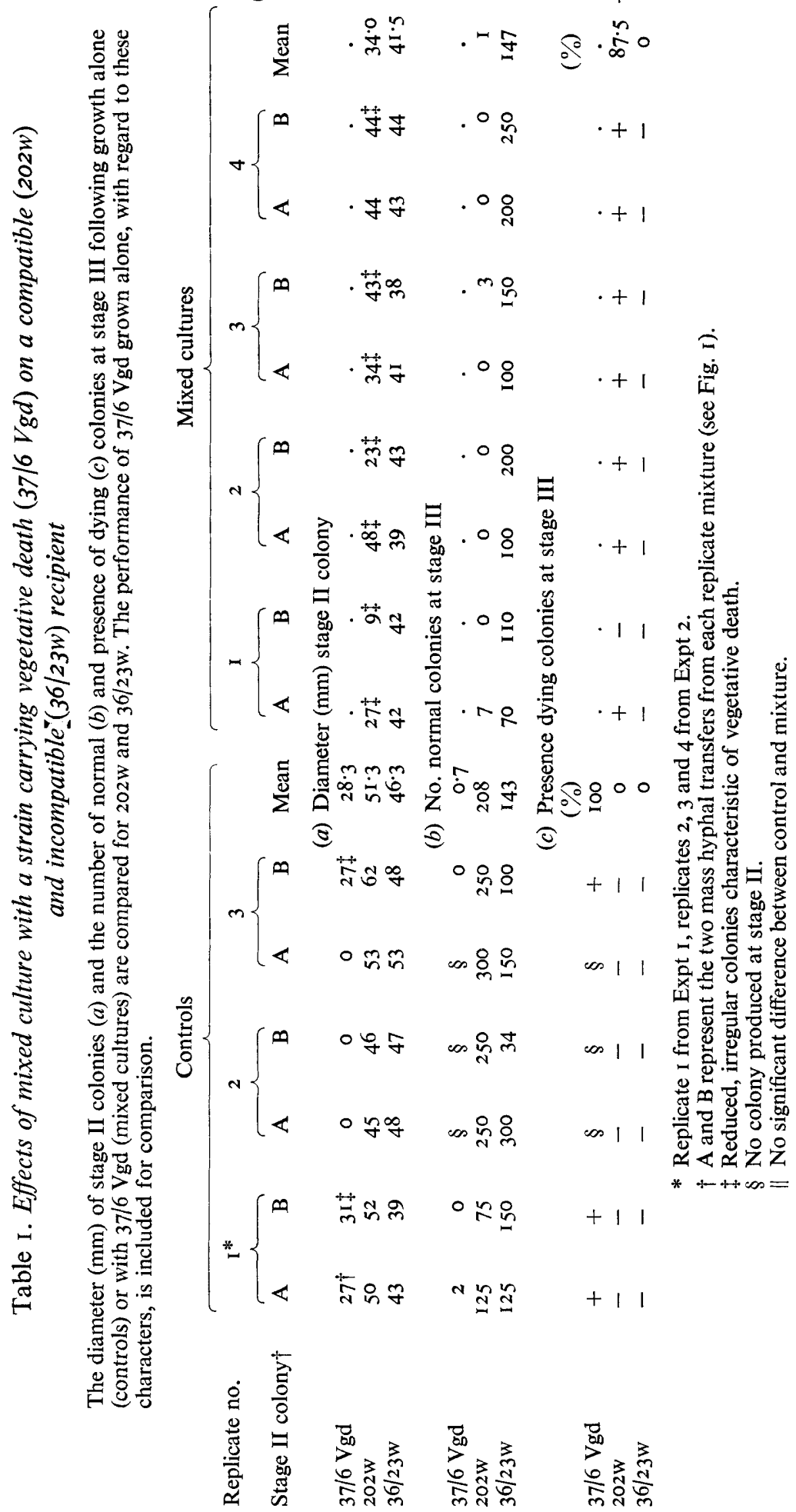


Table 2. Transfer of vegetative death to compatible and incompatible recipients

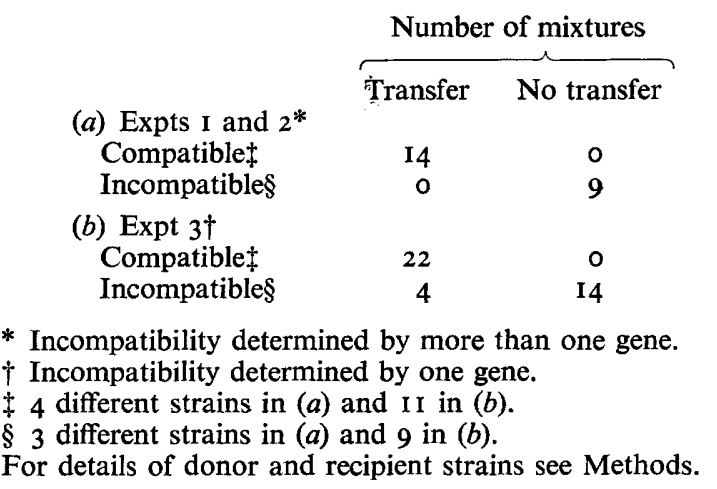

comparing the results for the 15 compatible and 12 incompatible strains of varying background genotype used. Vegetative death occurred spontaneously in the controls of two incompatible recipients in Expt 2, and all mixtures involving these strains were omitted from further consideraion. In Expts $I$ and 2 transfer of vegetative death occurred with I $00 \%$ success to compatible recipients but was completely blocked where the donor and recipient were incompatible (Table $2 a$ ). Restriction of cytoplasmic transfer by incompatibility was also apparent in Expt 3 (Table $2 b$ ), although it was no longer absolute. Considering the three experiments together cytoplasmic transfer occurred in all 36 compatible combinations examined but in only four of 27 (15\%) incompatible.

The four positive incompatible combinations involved different recipients and therefore the capacity to receive vegetative death from an incompatible donor does not appear to be strain specific. Despite their classification in Table 2 as transfer positive, the rate and extent of cytoplasmic infection in these four combinations was markedly less than in the compatible ones. Vegetative death was apparent at stage II in only two of the six colonies of incompatible recipients in which it was eventually revealed by the stage III platings, as opposed to 32 of 44 colonies of compatible recipients, in the same experiment. Similarly, whereas with the compatible combinations in all cases both mass hyphal samples from the same mixture developed vegetative death, this was so in only two of the four successful incompatible combinations.

\section{DISCUSSION}

The results presented show a clear association between compatibility and successful transfer of vegetative death on the one hand and incompatibility and failure of transfer on the other. Since both the compatible and incompatible recipients represented a variety of background genotypes, and in Expt 3 were progeny from a single cross segregating for compatibility, this association is most unlikely to have arisen by chance, and hence suggests a direct causal connection between the ability of strains to form vegetative associations and the transmission of vegetative death. Assuming vegetative death to be representative of cytoplasmically located genetic determinants (including viruses), we may conclude that vegetative incompatibility markedly inhibits cytoplasmic as well as nuclear exchange between strains. It follows from the genetics of vegetative incompatibility (Garnjobst, I953, I955; Esser, I97I; A. C. Butcher, C. E. Caten and J. Croft, unpublished) that a number of genes exist in Neurospora crassa, Podospora anserina and Aspergillus spp. which can 
confer resistance to invasion by suppressive cytoplasmic determinants. That a single gene may determine resistance has been demonstrated in Expt 3 for the het $A$ locus in $A$. amstelodami, although the possibility that the observed resistant:susceptible segregation results from a closely linked gene cannot be ruled out. The occurrence of transfer to four recipients whose incompatibility was determined by a single gene (Table $2 b$ ) while no instances of transfer were detected where incompatibility was determined by several genes (Table $2 a$ ), suggests a degree of additivity in the action of the heterokaryon incompatibility genes.

The use of the highly suppressive condition, vegetative death, as the cytoplasmic marker will have minimized the inhibition of transfer by incompatibility, since any reduction in frequency as opposed to complete abolition will have been rapidly overcome. That such a quantitative effect occurs is suggested both by the delay in the appearance of symptoms and the discrepancy between replicate samples shown by the four positive incompatible mixtures.

The results suggest that vegetative incompatibility will markedly reduce the spread of suppressive, cytoplasmic genetic elements, including viruses, from strain to strain in nature. Heterozygosity for a single incompatibility gene is insufficient to completely prohibit cytoplasmic infection, however, and it is interesting in this regard that all pairs of incompatible wild isolates of Aspergillus nidulans and A. amstelodami analysed to date differ at two or more such loci (Jinks, Caten, Simchen \& Croft, 1966; A. C. Butcher, C. E. Caten and J. Croft, unpublished). The situation in natural populations appears therefore more like Expts I and 2 than Expt 3, in which case cytoplasmic genetic infection through hyphal anastomosis and heterokaryon formation should be completely blocked.

The above considerations raise the question of the biological role of vegetative incompatibility in fungi. It has generally been assumed, by analogy with sexual incompatibility systems, that vegetative incompatibility serves to regulate genetic variability by controlling heterokaryosis and parasexual recombination (Caten \& Jinks, 1966; Esser, 197I). However, such a role is not consistent with the demonstrated occurrence of similar vegetative incompatibility systems in association with very diverse sexual breeding systems (Caten, I971). Day (1970) and Caten (I97I) have suggested that vegetative incompatibility might serve to protect mycelia from invasion by suppressive cytoplasmic determinants following hyphal anastomosis, and that its role is therefore one of cellular defence against genetic infection. In demonstrating that vegetative incompatibility markedly reduces, if not prohibits, cytoplasmic transfer, the results reported strongly support this rôle. That incompatibility acts in this way in nature is suggested by the observations of Grente \& Sauret (1969a,b) on exclusive hypovirulence among strains of Endothia parasitica.

I thank Professor J. L. Jinks, F.R.S., for his advice and constructive criticism throughout this investigation, and Dr D. A. Smith for his guidance in the preparation of the manuscript. I am indebted to Miss F. Moffatt for excellent technical assistance.

\section{REFERENCES}

Banks, G. T., Buck, K. W., Chain, E. B., Himmelweit, F., Marks, J. E., Tyler, J. M., Hollings, M., LAST, F. T. \& Stone, O. M. (I968). Viruses in fungi and interferon stimulation. Nature, London 218, $542-545$.

Bertrand, H., McDougall, K. J. \& Pittenger, T. H. (1968). Somatic cell variation during uninterrupted growth of Neurospora crassa in continuous growth tubes. Journal of General Microbiology 50, 337-350.

Border, D. J., Buck, K. W., Chain, E. B., Kempson-Jones, G. F., Lhoas, P. \& Ratti, G. (1972). Viruses of Penicillium and Aspergillus species. Biochemical Journal 127, 4P-6P. 
CATEN, C. E. (197I). Heterokaryon incompatibility in imperfect species of Aspergillus. Heredity 26, 299-3I2.

CATEN, C. E. \& JiNKs, J. L. (I966). Heterokaryosis: its significance in wild homothallic Ascomycetes and Fungi Imperfecti. Transactions of the British Mycological Society 49, 8I-93.

DAY, P. R. (1970). The significance of genetic mechanisms in soil fungi. In Root Diseases and Soil-borne Pathogens. Edited by T. A. Toussoun, R. V. Bega \& P. E. Nelson. Berkeley: University of California Press.

EsSER, K. (197r). Breeding systems in fungi and their significance for genetic recombination. Molecular and General Genetics I10, 86-100.

Esser, K. \& Kuenen, R. (1967). Genetics of Fungi, Ist edn, pp. 98-I00. New York: Springer Verlag.

FINCHAM, J. R. S. \& DAY, P. R. (I97I). Fungal Genetics, 3rd edn, pp. 312-313. Oxford: Blackwell Scientific Publications.

GaRNJOBST, L. (1953). Genetic control of heterokaryosis in Neurospora crassa. American Journal of Botany 40, 607-614.

GaRNJOBST, L. (1955). Further analysis of the genetic control of heterokaryosis in Neurospora crassa. American Journal of Botany 42, 444-448.

GARNJOBST, L. \& Wilson, J. F. (1956). Heterokaryosis and protoplasmic incompatibility in Neurospora crassa. Proceedings of the National Academy of Sciences of the United States of America 42, 613-618.

GRENTE, J. \& SAURET, S. (I969a). L'hypovirulence exclusive, phénomène original en pathologie végétale. Compte rendu hebdomadaire des séances de l'Académie des sciences (Série D) 268, 2347-2350.

Grente, J. \& SAURET, S. (1969b). L' 'hypovirulence exclusive' est-elle contrôlée par des determinants cytoplasmiques? Compte rendu hebdomadaire des séances de l'Académie des sciences (Série D) 268, 3173-31 76.

HoluINGs, M. (1962). Viruses associated with a die-back disease of cultivated mushroom. Nature, London I96, 962-965.

Hollings, M. \& Stone, O. M. (1969). Viruses in fungi. Science Progress, Oxford 57, 371-391.

Holloway, B. W. (1955). Genetic control of heterocaryosis in Neurospora crassa. Genetics 40, I 1 7-I 29.

JINKS, J. L. (1956). Naturally occurring cytoplasmic changes in fungi. Comptes rendus des travaux du Laboratoire Carlsberg (Série physiologique) 26, 183-203.

JINKS, J. L. (1959). Lethal suppressive cytoplasms in aged clones of Aspergillus glaucus. Journal of General Microbiology 21, 397-409.

Jinks, J. L., Caten, C. E., Simchen, G. \& Croft, J. H. (I966). Heterokaryon incompatibility and variation in wild populations of Aspergillus nidulans. Heredity 21, 227-239.

Jones, D. A. (1965). Heterokaryon incompatibility in the Aspergillus glaucus link group. Heredity 20, 49-56.

LhoAs, P. (1971). Transmission of double stranded RNA viruses to a strain of Penicillum stoloniferum through heterokaryosis. Nature, London 230, 248-249.

Marcou, D. (196r). Notion de longévité et nature cytoplasmique du déterminant de la sénescence chez quelques champignons. Annales des sciences naturelles (Botanique) (Série 12) 2, 653-764. 